\title{
In memoriam. Carlos Iván Degregori Caso
}

(Lima, I945-Lima, 20II)

Carlos Iván Degregori, quien desde 2007 hizo parte del comité científico de la Revista Colombiana de Antropología, trabajó en sus últimos años como investigador del Instituto de Estudios Peruanos (IEP) en Lima. Entre sus innumerables trabajos se destacan los estudios sobre la violencia política, los procesos de construcción de memoria y el análisis del desarrollo histórico de la antropología en Perú. Si bien dedicó su vida a la investigación y producción académica, en su juventud se debatió entre los estudios literarios y la antropología. Sin embargo, el contexto de los años setenta en el Perú y América Latina, caracterizado por el compromiso y cambio social y la vida política lo llevaron a dedicarse a las ciencias sociales.

$\mathrm{Su}$ vida académica transcurrió en varias universidades. Su formación inició con la Licenciatura en Antropología Social en la Universidad de San Cristóbal de Huamanga (UNSCH), en Ayacucho, y después, en el Departamento de Antropología de Brandeis University (Boston, Estados Unidos). Posteriormente, realizó estudios de maestría en Literatura Peruana y Latinoamericana en la Universidad Nacional Mayor de San Marcos (UNMSM) y más tarde hizo el Doctorado en Antropología Cultural de la Universidad de Utrecht, Holanda. Además de haber sido investigador y en dos ocasiones director del IEP, fue profesor y director del Departamento de Antropología de la Facultad de Ciencias Sociales de la unMSM. Asimismo, se desempeñó como docente invitado en las universidades de Princeton, Columbia, Wisconsin, John Hopkins, Libre de Berlín y la Escuela de Altos Estudios de Ciencias Sociales de París. Perteneció al Instituto de Democracia y Derechos Humanos (IDEHPUCP), a la Latin American Studies Association (LASA), al South-South Exchange Programme for Research on the History of Development (Sephis) y al Comité de América Latina del Social Science Research Council (SSRC).

Durante sus años de formación en la Universidad de San Cristóbal de Huamanga (UNSCH) vivió de cerca el proceso del Partido Comunista del Perú-Sendero Luminoso (PCPSL), el cual, según Dregregori, siguió la receta maoísta sin comprender el contexto cultural, lingüístico y político de los pueblos andinos, que lo llevó a la lógica del "marxismo de manual". En su trabajo 
sobre el desarrollo de la antropología en Perú, Degregori criticó este tipo de marxismo, sobre el cual escribió que:

[...] se consume en la ideologización, oculta y deja de lado la investigación empírica, reemplazándola por la lectura reverencial, ni siquiera de los clásicos, sino de los manuales de marxismo de la Academia de Ciencias de la uRss, que al condensar supuestamente toda la verdad vuelven superflua la investigación. (Degregori \& Sandoval, 2007, p. 314)

Es así como gran parte de su obra se dedica a la comprensión de la violencia política en Perú entre ig80 y 2000, en las publicaciones de su autoría así como en aquellas en las que participó como editor o compilador. Al respecto, vale la pena mencionar su libro Qué difícil es ser Dios. El Partido Comunista del PerúSendero Luminoso y el conflicto armado interno en el Perú: I980I999 (20II), en el que retoma varias de sus investigaciones y da una nueva estructura a muchos de sus escritos. Este trabajo ofrece un recuento del momento y las condiciones en las que surge el PCPSL y analiza los complejos procesos que desencadenan la violencia política (la exclusión, la discriminación étnica y cultural, así como el racismo). Además, incorpora sus reflexiones como miembro de la Comisión de la Verdad y la Reconciliación (CVR), entre 2001 y 2003.

Como académico promovió la investigación y la formación de nuevos profesionales, al tiempo que criticó las reformas neoliberales a la universidad y analizó las consecuencias que estas tuvieron en los contenidos de los planes curriculares y en la calidad de la enseñanza. Al respecto, los trabajos Antropología y antropólogos en el Perú. La comunidad académica de ciencias sociales bajo la modernización neoliberal (2009) y Saberes periféricos. Ensayos sobre la antropología en América Latina (2008) plantean la necesidad de tener una visión comparada de la disciplina en el Perú y en América Latina, que considere tanto su historia institucional e intelectual como sus vaivenes teóricos y políticos. En este sentido, en No hay país más diverso: compendio de antropología peruana (2000) ofrece un panorama general de la antropología peruana y plantea que el aporte central de esta fue contribuir a la articulación nacional a través de la exploración de territorios ignotos. Recientemente, a raíz de su preocupación por cerrar las brechas entre las universidades de Lima y las de provincia, a través de la promoción de una educación intercultural, 
inclusiva y que combine saberes y conocimientos, hizo parte del desarrollo del sitio web Cholonautas (http://www.cholonautas. edu.pe).

Carlos Iván Degregori produjo una obra extensa en la que se descubre su interés por la política y la antropología y una preocupación constante por la producción de conocimiento crítico sobre el contexto propio, Perú, y sobre América Latina en general.

\section{SELECCIÓN DE TRABAJOS de Carlos Iván Degregori}

(20II). Qué difícil es ser Dios. El Partido Comunista del Perú-Sendero Luminoso y el conflicto armado interno en el Perú: I980-I999. Lima: Instituto de Estudios Peruanos.

(2010). El surgimiento de Sendero Luminoso. Ayacucho I969-I979: del movimiento por la gratuidad de la enseñanza al inicio de la lucha armada ( $3^{\mathrm{a}}$ ed.). Lima: Instituto de Estudios Peruanos.

(2009). Antropología y antropólogos en el Perú. La comunidad académica de ciencias sociales bajo la modernización neoliberal. Lima: Instituto de Estudios Peruanos, Clacso-Argentina. [En coautoría con Pablo Sandoval].

(2008). Saberes periféricos. Ensayos sobre la antropología en América Latina. Lima: Instituto de Estudios Peruanos. [Compilación con Pablo Sandoval].

(2008). Peru: A Missed Opportunity. En J. I. Domínguez \& M. Shifter (Eds.), Constructing Democratic Governance in Latin America ( ${ }^{\mathrm{a}} \mathrm{ed}$; pp. 264-284). Baltimore: The Johns Hopkins University Press.

(2007). El nacimiento de los otorongos: el Congreso de la República durante los gobiernos de Alberto Fujimori (1990-2000). Lima: Instituto de Estudios Peruanos. [Coeditor con Carlos Meléndez Guerrero].

(2007). La antropología en el Perú: del estudio del otro a la construcción de un nosotros diverso. Revista Colombiana de Antropología, 43, 299-334. [En coautoría con Pablo Sandoval].

(2006). Perú: problema agrario en debate. Sepia XI. Lima: Sepia. [Coeditor con Javier Escobal y Javier Iguiñez].

(2006). Los hijos de la guerra. Gobernabilidad: Revista de Sociedad y Estado, I, 83-Io6. [En coautoría con R. J. López]. 
(2005). Cultura y globalización. Lima: Universidad del Pacífico. [En coautoría con Gonzalo Portocarrero].

(2005). The Peru Reader: History, Culture, Politics ( $2^{\mathrm{a}}$ ed.). Durham: Duke University Press. [Coedición con Robin Kirk y Orin Starn].

(2004). Discursos y violencia política en Sendero Luminoso. En G. Sánchez Gómez \& E. Lair (Eds.), Violencias y estrategias colectivas en la región andina: Bolivia, Colombia, Ecuador, Perú y Venezuela (pp. I45-I74). Lima: IFEA, Grupo Editorial Norma.

(2004). Ilave: desafío de la gobernabilidad, la democracia participativa y la descentralización. Lima: Grupo Propuesta Ciudadana.

(2003). Jamás tan cerca arremetió lo lejos: memoria y violencia política en el Perú. Lima y Nueva York: Instituto de Estudios Peruanos, Social Science Research Council. [Coeditor con Elizabeth Jelin].

(2003). Comunidades locales y transnacionales. Cinco estudios de caso en el Perú. Lima: Instituto de Estudios Peruanos. [Editor]

(2003). Fujimori: The Vanishing of a Regime and the Challenge of Democratic Rebuilding. En I. Jorge \& M. Domínguez (Eds.), Constructing Democratic Governance in Latin America ( $2^{\text {a }}$ ed.; pp. 220-243). Baltimore, London: The Johns Hopkins University Press

(2003). Perú: identidad, nación y diversidad cultural. En P. Oliart (Ed.), Territorio, cultura e historia: materiales para la renovación de la enseñanza sobre la sociedad peruana (pp. 212-223). Lima: Instituto de Estudios Peruanos, Comisión de Promoción del Perú, Promperú, GTz.

(2002). Estados nacionales, etnicidad y democracia en América Latina. Osaka: Japan Center for Area Studies, National Museum of Ethnology. [Compilación con Mutsuo Yamada].

(200I). La enseñanza de la antropología en el Perú. Lima: Consorcio de Investigación Económica y Social, Instituto de Estudios Peruanos. [En coautoría con Pablo Sandoval y Javier Ávila].

(2000). La década de la antipolítica. Auge y huida de Alberto Fujimori y Vladimiro Montesinos. Lima: Instituto de Estudios Peruanos.

(2000). No hay país más diverso: compendio de antropología peruana. Lima: Instituto de Estudios Peruanos. [Editor]

(2000). Discurso y violencia política en Sendero Luminoso. Boletín del Instituto Francés de Estudios Andinos, 29 (3), 493-513.

(I999). Ethnicity and Democratic Governability in Latin America. Reflections from Two Central Andean Countries. En F. Agüero (Ed.), Fault Lines of Democracy in Post-Transition Latin America (pp. 203-236). Miami: North South Center Press. 
(1999). Pueblos indígenas y democracia en América Latina. En J. Nieto Montesinos (Comp.), Sociedades multiculturales y democracias en América Latina (pp. I77-210). México: Unesco, Unidad para la Cultura Democrática y la Gobernabilidad, Demos.

(I998). Government, Citizenship and Democracy: A Regional Perspective. En J. Crabtree \& J. Thomas (Eds.), Fujimori’s Peru: The Political Economy (pp. 437-465). Londres: University of London. Institute of Latin American Studies. [En coautoría con José Coronel Aguirre y Ponciano del Pino H.].

(I998). Movimientos étnicos, democracia y nación en Perú y Bolivia. En C. Dray (Ed.), La construcción de la nación y la representación ciudadana en México, Guatemala, Perú, Ecuador y Bolivia. Guatemala: Flacso.

(1998). Harvesting Storms. Peasant Rondas and the Defeat of Shining Path in Ayacucho. En S. Stern (Ed.), Shining and Other Paths. War and Society in Peru, I980-1995 (pp. I28-I57). Durham: Duke University Press.

(1997). After the Fall of Abimael Guzmán. The Limits of Sendero Luminoso. En M. Cameron \& P. Mauceri (Eds.), The Peruvian Labyrinth. Polity, Society, Economy (pp. I79-I9I). University Park: Pennsylvania State University Press.

(1997). The Maturation of a Cosmocrat and the Building of a Discourse Community: The Case of Shining Path. En D. Apter (Ed.), The Legitimation of Violence (pp.33-82) London: Unrisd, MacMillan Press.

(I996). Las rondas campesinas y la derrota de Sendero Luminoso. Lima: Instituto de Estudios Peruanos.

(I996). Perú: crisis del régimen constitucional en un contexto de ajuste neoliberal y violencia política. En P. Gaitán Pavia, R. Peñaranda, E. Pizarro Leongómez (Comps.), Democracia y reestructuración económica en América Latina (pp. 208-224). Bogotá: Cerec, Iepri.

(1995). The Peru Reader. History, Culture, Politics. Durham y London: Duke University Press. [Coeditor con Orin Starn y Robin Kirk].

(1994). Shining Path and Counterinsurgency Strategy since the Arrest of Abimael Guzmán. En J. Tulchin \& G. Bland (Eds.), Peru in Crisis. Dictatorship or Democracy? (pp. 8I-IOO). Washington D. C.: Wilson Center.

(1994). Perou: l' effondrement surprenant du Sentier Lumineux. Problemes d'Amerique Latine, I3, 3-I7.

(1991). Elecciones I990. Demonios y redentores en el Nuevo Perú. Una tragedia en dos vueltas. Lima: Instituto de Estudios Peruanos. [Coeditor con R. Grompone]. 
(I99I). How Difficult It Is To Be God. Critique of Anthropology, II (3), 233-250.

(I99I). Jóvenes y campesinos ante la violencia política: Ayacucho I980I983. En H. Urbano \& M. Lauer (Comps.), Poder y violencia en los Andes (pp. 395-4I7). Cusco: Centro de Estudios Regionales Andinos Bartolomé de Las Casas.

(I99I). The Origins and Logic of Shining Path. Return to the Past. En D. S. Palmer (Ed.), Shining Path Of Peru (pp. 38-44). New York: St. Martin's Press.

(I990). Tiempos de ira y amor. Lima: Desco.

(I989). Qué difícil es ser Dios. Ideología y violencia política en Sendero Luminoso. Lima: El Zorro de Abajo Ediciones.

(I989). El desafío de Huayopampa: comuneros y empresarios ( ${ }^{\text {a }}$ ed.). Lima: Instituto de Estudios Peruanos. [Coeditor con Fernando Fuenzalida y otros].

(I986). Ayacucho: raíces de una crisis. Ayacucho: Instituto de Estudios Regionales José María Arguedas.

(I986). Conquistadores de un nuevo mundo: de invasores a ciudadanos en San Martín de Porres. Lima: Instituto de Estudios Peruanos. [En coautoría con Cecilia Blondet y Nicolás Lynch].

(I985). Sendero Luminoso: los hondos y mortales desencuentros. Lima: Instituto de Estudios Peruanos.

(1978). Indigenismo, clases sociales y problema nacional: la discusión sobre el "problema indígena" en el Perú. Lima: Centro Latinoamericano de Trabajo Social. [Editor]

(I967). Cambios estructurales y limitaciones ecológicas: proceso histórico de la comunidad de Santa Lucía de Pacaraos. Lima: Instituto de Estudios Peruanos, Cornell University, unMsm. Facultad de Letras. Departamento de Antropología. [En coatoría con Jürgen Golte, Modesto Gálvez R. y Jaime Urrutia Ceruti].

SARAh NiEto

Asistente editorial Revista Colombiana de Antropología 